\title{
Advanced numerical models for the propagation of floods with high-sediment concentrations in mountain rivers
}

\author{
María Teresa Contreras ${ }^{1,2,3}$, Jorge Gironás ${ }^{1,2}$, Joannes Westerink $^{3}$, and Cristián \\ Escauriaza $^{1,2, *}$ \\ ${ }^{1}$ Departamento de Ingeniería Hidráulica y Ambiental, Pontificia Universidad Católica de Chile, Av. \\ Vicuña Mackenna 4860, Santiago, 7820436, Chile. \\ ${ }^{2}$ Centro de Investigación para la Gestión de Desastres Naturales (CIGIDEN) \\ ${ }^{3}$ Department of Civil and Environmental Engineering and Earth Sciences, University of Notre Dame, \\ USA
}

\begin{abstract}
Rapid floods induced by extreme precipitation are common events in regions near the Andes mountain range. Growing urban development, combined with the changing climate and the influence of El Niño, have increased the exposure of the population in many regions of South America. Simulations of flash floods in these watersheds are very challenging, due to the complex morphology, the insufficient hydrometeorological data, and the uncertainty posed by the variability of sediment concentration. To address these issues, we develop a high-resolution numerical model of the non-linear shallow water equations, coupled with the mass conservation of sediment, and considering the density effects and changes of rheology in the momentum equation. Based on these simulations we develop a real-time early-warning system, by creating a surrogate model or meta-model from the simulations. Using a small set of parameters, we define storms for a wide range of meteorological conditions, and utilize the high-fidelity model results to create a database of flood propagation under different conditions. Through this second model we perform a sophisticated interpolation/regression, and approximate efficiently the flow depths and velocities. This is the first application of its kind in the Andes region, which can be used to improve the prediction of flood hazard in real conditions, employing low computational resources. We also create a framework to develop early warning systems, and to help decision makers and city planners in these mountain regions.
\end{abstract}

\section{Introduction}

Flash floods produced by extreme precipitation events have shown to frequently affect cities and infrastructure in mountain regions. In many cases, anthropic factors such as unplanned urban development and climate change can amplify significantly their consequences, increasing the risk for the population and affecting their social and economical conditions [1]. The Andes mountains in South America, for example, have been the scenario of many recent

\footnotetext{
*e-mail: cescauri@ing.puc.cl
} 
floods with catastrophic outcomes, and the piedmont has experienced a rapid urban growth, occupying regions near river channels, and increasing the exposure of the communities and their infrastructure.

To assess flood hazards and design strategies to reduce the potential damages caused by flooding, we need to improve our understanding on the controlling mechanisms that determine the propagation of the floods in mountain streams. These regions, however, are characterized by a lack of accurate field information, and by short records of hydrometeorological data.

Therefore, numerical models can play a significant role on the prediction of these events and the evaluation of potential scenarios. Models are now becoming essential tools to understand the propagation and consequences of floods, plan the future adaptation of cities affected by natural hazards, and develop early warning systems in urban areas.

In spite of the recent advances of numerical models used to predict the dynamics of floods, mountain rivers present additional challenges, due to the complex bathymetries and steep slopes of the river channels that produce abrupt changes on velocities and water depths, and large sediment concentrations that induce additional stresses altering the mass and momentum balance of the flow. In addition, high-resolution models might have significant computational costs, which can prevent the timely prediction of the flood propagation necessary for early warning systems, especially when the uncertainty of the flood conditions requires the evaluation of multiple possible scenarios of the event.

In this investigation we develop two models for studying the propagation of rapid floods in an Andean watershed: (1) We implement a two-dimensional high-resolution numerical model of the shallow-water equations coupled to a hydrological model, to simulate the flood dynamics in complex arbitrary geometries, considering also the effects of large sediment concentrations; and (2) We develop a surrogate model based on the results of the high-fidelity approach, which can rapidly calculate new scenarios characterized by a set of parameters that define the hydrometeorological events, using a statistical interpolation in the parameter space.

The high-fidelity model can capture the dynamic interplay between the high sediment concentrations and geomorphic drivers on the flood propagation in mountain streams, revealing the most important physical aspects of the flow. The surrogate model, on the other hand, provides high-resolution results with low computational costs, using a database of cases that are precomputed with the $2 \mathrm{D}$ model for the fast evaluation of multiple scenarios, which can be used for developing early warning systems.

The paper is organized as follows: In section 2 we explain the characteristics that define extreme events in Andean watersheds, which is applied to the Quebrada de Ramón stream near Santiago, Chile. Section 3 presents the high-fidelity 2D model that we utilize to simulate the propagation of floods with high sediment concentrations. In section 4 we describe a new surrogate model or meta-model for early warning systems, which employs the database of simulations with the numerical model for different possible scenarios. Finally, section 5 contains the conclusions, the summary of the findings of our research, and future directions of study.

\section{Modeling Floods in Mountain Rivers}

The development of efficient and accurate numerical models to simulate flash floods is far from trivial, since multiple factors control the dynamics of the flow. Especially in mountainous regions, where rivers are characterized by complex bathymetries, lack of data in highaltitude environments, difficult access, extreme weather conditions, and large sediment concentrations. 
The proximity of river channels to urban areas, and the lack of monitoring are some of the factors that increase the flood hazards in the region. In addition, storms that are influenced by El Niño Southern-Oscillation (ENSO) cause significant rainfall with warm temperatures, elevating the $0^{\circ} \mathrm{C}$ isotherm. These warm conditions generate a large total contributing area of liquid precipitation, increasing the runoff volume, the peak discharges, and producing high flow velocities and sediment transport in the flow.

High sediment concentrations during floods cause additional stresses produced by the increase of density and viscosity of the water-sediment mixture. In cases with homogeneous fluids and no significant sediment transport, numerical models only consider a flow resistance term due to the bed shear stresses, represented by coefficients derived for uniform flows. In hyperconcentrated flows, however, models need to account for the internal stresses that emerge from the particle-flow and particle-particle interactions.

To understand the effects of hydrometeorological events and sediment concentration on the flood hydrodynamics in the Andes, we select the Quebrada de Ramón watershed as the case of study. This basin has a total area of $38.5 \mathrm{~km}^{2}$ and it is located in central Chile, to the east of the city of Santiago. In the lower section of the watershed, the natural channel has been modified and diverted into a concrete channel with a design discharge of $20 \mathrm{~m}^{3} / \mathrm{s}$. The size of the basin and the range of elevations ( 800 to $3400 \mathrm{~m}$ a.s.l.) generate high velocities and hyperconcentrated flows during flood events, and the hydrological response of the catchment is very sensitive to the location of the $0^{\circ} \mathrm{C}$ isotherm elevation, and small variations of the freezing level position originate large differences on the contributing area.

In the following sections we present the models we implement in this investigation. For the hydrodynamic model to simulate the propagation of floods we use the nonlinear shallow water equations (NSWE) or Saint Venant equations. They are obtained by vertically averaging the three-dimensional Navier-Stokes equations, assuming a hydrostatic pressure distribution, resulting in a set of horizontal two-dimensional (2D) hyperbolic conservation laws that describe the evolution of the water depth and depth-averaged velocities in space and time. In this case we also incorporate the effects of hyperconcentration in mass and momentum by coupling the system of equations to the conservation equation of sediment concentration. The surrogate model uses the results of this hydrodynamic model to build a statistical interpolation, and provide a fast response for different scenarios that have not been simulated.

\section{High-Fidelity Simulations}

The analysis of flood events requires the definition of the meteorological event, and the natural characteristics of the watershed that explain the evaporation, storage, and infiltration processes at the headwaters. For example, extreme precipitation events with high temperatures in the Andes generate a large volume of surface water, which is rapidly propagated due to the steep slopes in the mountains. The high-fidelity approach we describe in this section couples a semi-distributed hydrological model to with a high-resolution hydrodynamic model in the main channel. These simulations can capture the effects of the floods in the city, determining the evolution of the instantaneous velocity and flow depth.

\subsection{Hydrological modeling}

To estimate the discharge hydrograph, we implement a continuous hydrological model using the Hec-HMS model [2], which represents the rainfall-runoff process by considering a continuous record of meteorological data. Using an hourly record of precipitation spanning 
40 years, we perform an statistical disaggregation in time to generate a series of precipitation events in the watershed, producing a synthetic record for a total of 200 years. From the hydrometeorological perspective, the distribution and volume of precipitation is the critical factor in the magnitude of the flood, which in turns depends on the spatial and temporal variation of air temperature, and the elevation of the $0^{\circ} \mathrm{C}$ isotherm.

For the hydrological model we divide the watershed in 12 subcatchments, and obtained their morphological metrics from a digital elevation model (DEM) with a resolution of 2 $\mathrm{m}$. To produce the hydrographs, the model considers the storage, infiltration, interceptions, evapotranspiration, and snow accumulation and melting.

\subsection{Hydrodynamic modeling}

We solve the non-linear shallow-water equations (NSWE), which correspond to the conservation of mass and momentum, assuming hydrostatic pressure distribution. Since the high sediment concentrations can change significantly the rheology of the flow, we modify the equations to account for the heterogeneous density distribution. In the numerical solution we compute the evolution in time and space of the flow depth $h$, the sediment concentration $C$, and the cartesian components of the 2D flow $u$ and $v$ in the $X$ and $Y$ directions, respectively.

To adapt the computational domain to the complex arbitrary topography in mountainous watersheds, we use a boundary fitted curvilinear coordinate system, denoted by the coordinates $(\xi, \eta)$. Through this transformation we can have a better resolution in zones of interest and an accurate representation of the boundaries. We perform a partial transformation of the equations, and write the set of dimensionless equations in vector form as follows,

$$
\frac{\partial Q}{\partial t}+J \frac{\partial F}{\partial \xi}+J \frac{\partial G}{\partial \eta}=S_{b}(Q)+S_{S}(Q)+S_{C}(Q)
$$

where $Q$ is the vector that contains the non-dimensional cartesian components of the conservative variables $h, h u, h v$ and $h C$, which are obtained by replacing the density of the mixture $\rho=C \rho_{s}+(1-C) \rho_{w}$, where $\rho_{w}$ is the water density, and $\rho_{s}$ the sediment density.

The Jacobian of the coordinate transformation $J$ is expressed in terms of the metrics $\xi_{x}$, $\xi_{y}, \eta_{x}$ and $\eta_{y}$, such that $J=\xi_{x} \eta_{y}-\xi_{y} \eta_{x}$. The fluxes $F$ and $G$ in each coordinate direction expressed as follows,

$$
F=\frac{1}{J}\left(\begin{array}{c}
h U^{1} \\
u h U^{1}+\frac{1}{2 F^{2}{ }^{2}} h^{2} \xi_{x} \\
v h U^{1}+\frac{1}{2 F^{2}} h^{2} \xi_{y} \\
C h U^{1}
\end{array}\right), G=\frac{1}{J}\left(\begin{array}{c}
h U^{2} \\
u h U^{2}+\frac{1}{2 r^{2}} h^{2} \eta_{x} \\
v h U^{2}+\frac{1}{2 F r^{2}} h^{2} \eta_{y} \\
C h U^{2}
\end{array}\right)
$$

where $U^{1}$ y $U^{2}$ represent the contravariant velocity components defined as $U^{1}=u \xi_{x}+v \xi_{y}$ and $U^{2}=u \eta_{x}+v \eta_{y}$, respectively.

The model considers three source terms: $S_{B}$ contains the bed slope terms, $S_{S}$ corresponds to the bed and internal stresses of the flow, and $S_{C}$ incorporates the effects of gradients of sediment concentration.

In the present numerical model we modify the quadratic model of O'Brien and Julien (1985) [3] to represent better the stresses for a wide range of sediment concentrations, expressing clearly the contribution of each physical mechanism as the combination of relations that account for the stresses, which have been obtained from experiments or physically-based formulas. We modify the model, however, to converge to the classical flow resistance formulas when the concentration is zero. The source terms for the bed and internal stresses for the $i$ coordinate direction are expressed as:

$$
S_{S i}=S_{\text {yield }}+S_{v_{i}}+S_{t d_{i}}
$$


where $S_{\text {yield }}$ represents the sum of the yield and Mohr-Coulomb stress, $S_{v_{i}}$ the viscous stress and $S_{t d_{i}}$ the sum of the dispersive and turbulent stresses.

The numerical solution of the system of equations is based on the method developed by Guerra et al. (2014) [4] to solve the NSWE, which has shown great efficiency and precision to simulate extreme flows and rapid flooding over natural terrains and complex geometries. This is a finite-volume formulation that is implemented in two steps: First, in the so-called hyperbolic step, the Riemann problem is solved at each element of the discretization without considering momentum sinks. The flow is reconstructed hydrostatically from the bed slope source-term, adding the effects of the spatial concentration gradients. In the second step we incorporate the shear stress source terms by means of a semi-implicit scheme, correcting the predicted values of the hydrodynamic variables. To compute the numerical fluxes we implement the VF-Roe-NCV method, linearizing the Riemann problem [4]. The MUSCL scheme is used to perform the extrapolation with second order accuracy in space. The model has been validated for many cases including supercritical flows and wave propagation on dry surfaces, comparing also the results with analytical solutions and experiments that include sharp density gradients [5].

For the Quebrada de Ramón stream, we simulate different cases with resolutions of 0.5 $\mathrm{m}$ near the main channel. Figure 1 shows the instantaneous results of the simulations for a peak flow of $97 \mathrm{~m}^{3} / \mathrm{s}$ with $38 \%$ of sediment concentration. The resolution of the simulations in time and space shows the evolution of the flow in the city, and the regions where the sediment concentration produces significant changes on the velocity and flow depth. We have also performed a complete analysis on the effects of hyperconcentration on the hydrograph, velocity of the wave front, evaluating also the effects of high sediment concentrations and geomorphic drivers on the propagation of floods in mountain streams [5].

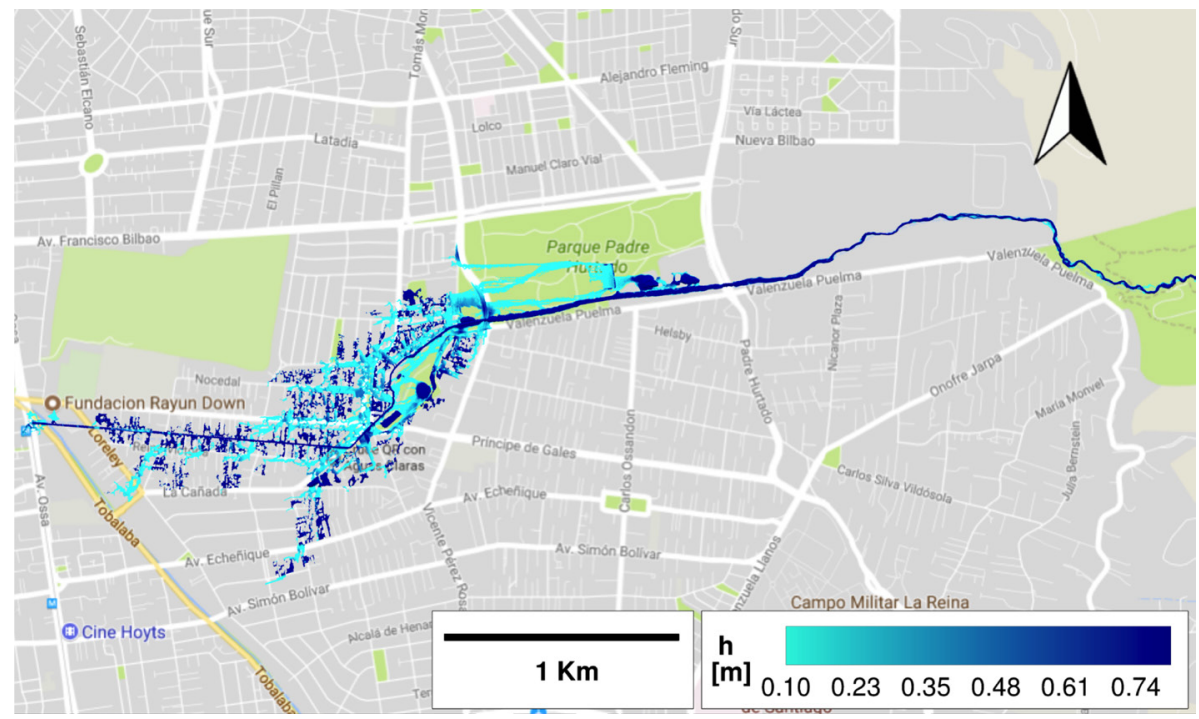

Figure 1. Instantaneous flow depth in the Quebrada de Ramón and the flooded area in Santiago, Chile, for a peak flow of $97 \mathrm{~m}^{3} / \mathrm{s}$ and $38 \%$ of sediment concentration. 


\section{Surrogate Model}

Simulations with the coupled hydrological and hydrodynamic models have high computational costs, especially if many scenarios need to be evaluated. An efficient implementation of early warning systems might rely on surrogate models, which can yield a fast response and provide information of the flow under different possible scenarios.

The implementation of early warning systems is now critical to manage flood risks in cities located near mountain rivers. A combination of predictive models with physical and statistical approaches are required to issue a warning, and take the necessary action for protecting human lives and property. Our limited understanding of these natural systems has prompted the development of a combination of high-resolution approaches, as the model presented in the previous section, and statistical models that can use a database of simulations to provide fast scenarios. In this section we describe the characterization of events to generate a database of precomputed scenarios, and an example of the surrogate formulation in the Quebrada de Ramón stream.

\subsection{Database generation}

To develop the surrogate model we need to generate a database of cases simulated with the high-fidelity model. The main advantage of this statistical approach is to produce fast scenarios for decision makers, obtained directly from the characteristics of the meteorological event without employing the high-fidelity approach, which requires running the hydrological and hydrodynamic models.

In the first step, we define the scenarios that will be precomputed to generate the database. To obtain a fast answer of the hydrodynamic variables in the city, we characterize the event using variables that can be obtained from the meteorological forecast.

We perform a simplified description of floods scenarios through a small number of model parameters based on the storm characteristics, incorporating also the effects of the sediment concentration. In this framework, any flood event can be characterized by only four parameters:

1. Mean of the precipitation event, which contains information of the intensity of rainfall;

2. Second statistical moment of the precipitation, to incorporate the temporal distribution of the event;

3. Minimum temperature of the event, which yields information on the elevation of the $0^{\circ} \mathrm{C}$ isotherm; and

4. Sediment concentration to consider the effects of the rheology, and capture the changes of velocity and depth when the concentration is high.

Initially we simulate 58 scenarios that are selected by using a latin hypercube sampling of cases, varying the magnitude of these parameters within realistic ranges.

\subsection{Results examples}

The database of simulations is used to fit the surrogate model [6]. To determine flow depths and velocities at specific locations in the city in new scenarios, we use an ordinary Kriging interpolation in the parameter space, considering a Gaussian correlation model among the 4 parameters to compute the covariance matrix that defines the dependence structure [6-8], and assuming a zero-order base regression for the hydrodynamic variables. 
Since the channel in the urban area has a maximum capacity of $20 \mathrm{~m}^{3} / \mathrm{s}$, we evaluate scenarios at the exit of this channel that can exceed the maximum flow. In Figure 2 we show the discharge at this specific point in the watershed, calculated for different scenarios that have not been simulated with the high-fidelity approach. The magnitude of the flow is consistent with the physical processes described for this basin, as high temperature events can produce significant floods for moderate average precipitation events.

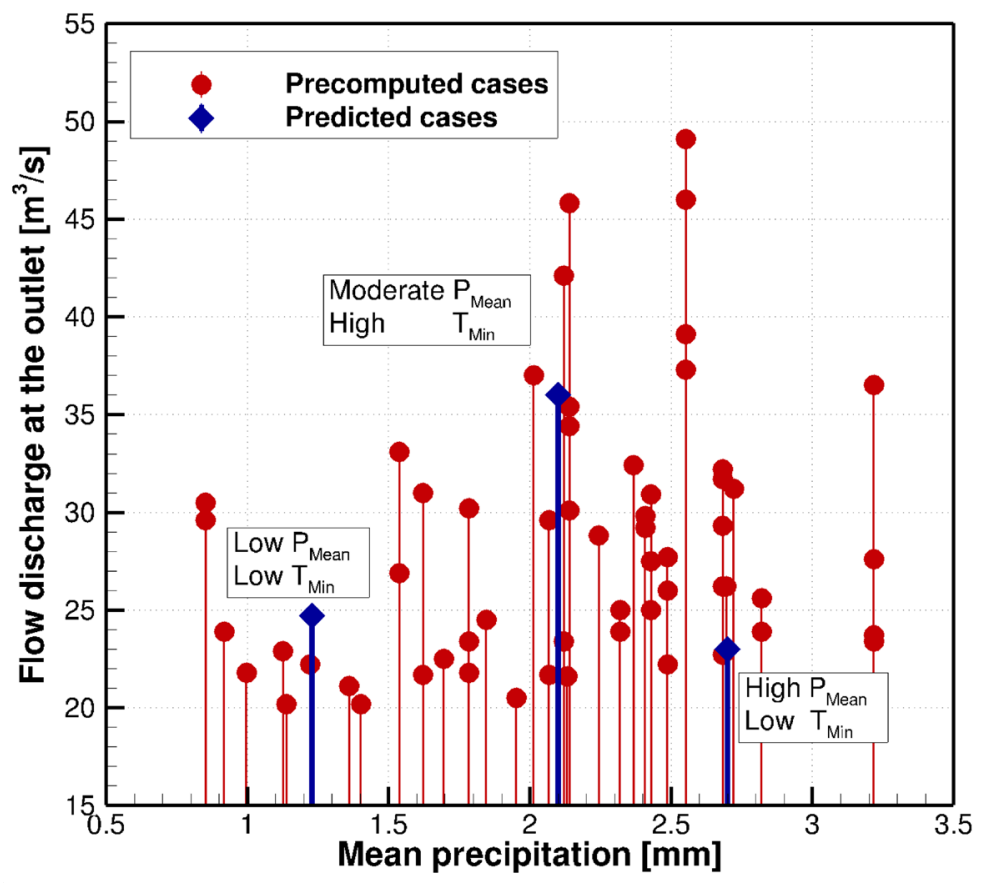

Figure 2. Results of the surrogate model for the flow discharge, compared to the data obtained from the high-fidelity approach.

\section{Conclusions}

The simulation of flood hazards by extreme precipitation in mountain streams requires numerical models capable of capturing complex flows that are influenced by the geomorphic features of the channel, and by high sediment concentrations that are common in these regions. In this investigation we develop two models for simulating the flow in an Andean watershed in central Chile: (1) A hydrologic model combined with a 2D hydrodynamic model that is coupled with the sediment concentration in mass and momentum; and (2) A surrogate model that employs precalculated scenarios of the previous models, to interpolate new cases using Kriging.

The statistical model utilizes low computational resources, and it is ideally suited for early warning systems as it can provide a fast response and evaluate different scenarios. To develop this surrogate model, we characterize the extreme flood events by statistical parameters of the storm, and by the sediment concentration. From a database of simulations, we fit a correlation model and perform Kriging to obtain hydrodynamic variables at any point in the domain. 
In the future, we expect that this model will be incorporated in an automated framework, to provide an advanced tool for decision makers and stakeholders, who can evaluate scenarios without the technical expertise on the calculation of complex flows during extreme hydrometeorological events. However, two important issues need to be resolved in our future research. First, we will increase the database of high-fidelity simulations and perform a full validation study of the surrogate model, testing also a deterministic regression combined with the interpolation to improve the predictions. The second improvement will be the analysis of the entire urban area. The current surrogate model yields the hydrodynamic variables at specific critical points within the city. In future research we will provide a complete analysis of the urban area DEM, which is important when we evaluate flood risk in the context of other hazards that exist in the city.

\section{Acknowledgements}

This work has been supported by CONICYT/FONDAP grant 15110017, and by the Vice Chancellor of Research of the Pontificia Universidad Católica de Chile, through the Research Internationalization Grant, PUC1566 funded by MINEDUC.

\section{References}

[1] Wohl, E. Inland Flood Hazards: Human, Riparian, and Aquatic Communities (Cambridge University Press, NY, 2011) 518 p.

[2] U.S. Army Corps of Engineers. HEC-HMS. User's Manual (version 4.1, USACE, 2015) $148 \mathrm{p}$.

[3] O'Brien, J., and Julien, P. Physical properties and mechanics of hyper-concentrated sediment flows (Proc. ASCE HD Delineation of landslides, flash flood and debris flow hazards, 1985) 260-279.

[4] Guerra, M., Cienfuegos, R., Escauriaza, C., Marche, F., and Galaz, J. Journal of Hydraulic Engineering, 140, (2014).

[5] Contreras, M. T. and Escauriaza, C. submitted to Adv. Water Res., (2018).

[6] Taflanidis, A. A., Kennedy, A. B., Westerink, J. J. et al. Journal of Waterway, Port, Coastal, and Ocean Enginering, 139, 171-182, (2013)

[7] Forrester, A. I. J., Sóbester, A., and Keane, A. J., Engineering Design via Surrogate Modelling (Wiley, UK, 2008) 210 p.

[8] Lophaven, S. N., Nielsen, H. B., and Sondergaard, J. DACE: A Matlab Kriging toolbox, version 2.0. (IMM Technical University of Denmark, Lyngby, 2002). 